\title{
Ultrasound-guided truncal blocks for perioperative analgesia
}

Received March 7, 2018

Revised April 2, 2018

Accepted April 3, 2018

\section{Corresponding author}

Won Uk Koh, M.D., Ph.D. Department of Anesthesiology and Pain Medicine, Asan Medical Center, University of Ulsan College of Medicine, 88 Olympic-ro 43-gil, Songpa-gu, Seoul 05505, Korea Tel: 82-2-3010-3868

Fax: 82-2-3010-6790

E-mail: koh9726@naver.com ORCID

http://orcid.org/0000-0003-4881-1884

\section{Won Uk Koh and Jong Hyuk Lee}

Department of Anesthesiology and Pain Medicine, Asan Medical Center, University of Ulsan College of Medicine, Seoul, Korea
The widespread application of ultrasound in clinical anesthesiology has led to an increase in the number of practitioners performing peripheral nerve blocks of the trunk to produce analgesia and anesthesia for surgeries involving the thorax, abdomen, and lower extremities. The use of real-time ultrasound has allowed the peripheral nerves, planes, and plexuses of the trunk to be located more accurately and has improved the success rate of blocks. Compared to central neuraxial techniques, many types of truncal blocks are relatively easy to perform and have low side effect profiles. As a result, truncal blocks have been introduced to provide perioperative analgesia for surgeries involving the thorax and abdomen. The most frequently used truncal block techniques include the paravertebral block, intercostal block, pectoralis and serratus anterior block, rectus sheath block, transversus abdominis plane block, and ilioinguinal and iliohypogastric nerve blocks. Recently introduced techniques also include the quadratus lumborum and erector spinae plane blocks. To apply each block correctly and reduce the likelihood of related side effects and complications, the practitioner must have a thorough understanding of the anatomical region, optimal block positioning, and device selection and management.

Key Words: Analgesia, Nerve block, Trunk, Ultrasonography.

\section{INTRODUCTION}

Since the widespread introduction of ultrasound for assisting techniques used in anesthetic theatres, peripheral nerve blocks of the trunk have been used more frequently to produce analgesia and anesthesia for surgeries involving the thorax, abdomen, and lower extremities. The introduction of real-time ultrasound has allowed the peripheral nerves, planes, and plexuses of the trunk to be located more accurately and has improved the success rate of truncal blocks [1]. Compared to traditional techniques, ultrasound-guided peripheral nerve block procedures enable anesthesiologists to reliably inject local anesthetic (LA) at a target location with a decreased risk of needle trauma to the nerve and surrounding structures. Furthermore, numerous studies have demonstrated that the effectiveness of perioperative pain management using peripheral nerve blocks of the trunk is comparable to that using traditional central neuraxial techniques [2-6]. Many types of truncal blocks have been described and introduced. The most frequently used techniques include the paravertebral block (PVB), intercostal block, pectoralis (Pecs) and serratus anterior block, rectus sheath block, transversus abdominis plane (TAP) block, and ilioinguinal and iliohypogastric nerve blocks. Recently introduced techniques also include the quadratus lumborum (QL) and erector spinae plane (ESP) blocks.

The purpose of this review is to introduce the various truncal blocks used for abdominal and thoracic surgeries, and to

This is an Open Access article distributed under the terms of the Creative Commons Attribution Non-Commercial License (http://creativecommons.org/licenses/by-nc/4.0) which permits unrestricted non-commercial use, distribution, and reproduction in any medium, provided the original work is properly cited. 
describe the scientific basis of each block. A brief overview of the technical aspects associated with commonly performed truncal blocks is also provided.

\section{THORACIC PVB}

\section{Clinical background and anatomy}

The PVB was first described in 1905 by Hugo Sellheim for abdominal analgesia [7]. After its introduction, efforts were made to apply the technique for abdominal and thoracic surgeries. Several ultrasound-guided techniques have been developed since 2009 [8-11] and although the basic procedure is still widely used [12], these ultrasound-guided techniques are now being used more frequently. The thoracic paravertebral space includes the thoracic spinal nerves, which emerge from the medial side of this space as they exit the thoracic intervertebral foramen [13]. The thoracic paravertebral space is triangular in transverse section with the medial base formed by the posterolateral aspect of the vertebral body, articular process, and intervertebral foramen $[7,13,14]$. The anterolateral border is formed by the parietal pleura; and the posterior border is formed by the transverse process, heads of ribs, and superior costotransverse ligament $[7,13,14]$. The paravertebral space communicates laterally with the intercostal spaces and medially with the epidural space through the intervertebral foramen. It contains the thoracic spinal nerve roots, thoracic sympathetic chain, ramus communicans, intercostal vessels, and fat tissue. Therefore, depositing LA into this space induces a blockade of the ipsilateral somatosensory and sympathetic nerves, which makes the PVB an effective modality for anesthesia and analgesia of pain originating from the chest and abdomen. Furthermore, the continuous communication among the thoracic paravertebral space, upper segment levels, and adjacent intercostal and epidural spaces allows a single injected bolus of LA to reach several adjacent dermatomes, although the level of spread may vary [15].

\section{Ultrasound-guided thoracic PVB techniques}

Various ultrasound-guided approaches have been described that target the thoracic paravertebral space. These can be categorized as approaches that use either transverse or parasagittal ultrasound images, needle entry in the lateral- to-medial or medial-to-lateral trajectory, and in-plane or outof-plane techniques [16]. However, in all of these techniques, the most important landmark structures in the ultrasound image are the transverse process of the thoracic spine, rib, and pleura. In this section, we consider ultrasound-guided thoracic PVB techniques using: the transverse cross-section in-plane approach in the lateral to medial trajectory; and the parasagittal cross-section in-plane approach in the caudal to cranial trajectory.

\section{Transverse approach}

For the transverse approach, the ultrasound transducer is positioned transversely to obtain an axial view of the trunk lateral to the midline in the ipsilateral direction. A highfrequency linear probe is used to generate the ultrasound image. Initially, an image of the bony structures including the spinous process, transverse process, and rib at the target vertebral level is obtained using ultrasound. Next, the ultrasound probe is moved slightly cephalad so that the rib disappears, and an image showing the transverse process and pleura is obtained (Fig. 1). A needle is inserted in the lateral to medial direction so that the needle tip enters the paravertebral space under the transverse process and above the pleura (Supplementary Image 1). Care must be taken to avoid penetrating the pleura or entering the epidural or intrathecal space. A single dose of 10-20 ml LA is injected, and a continuous cath-

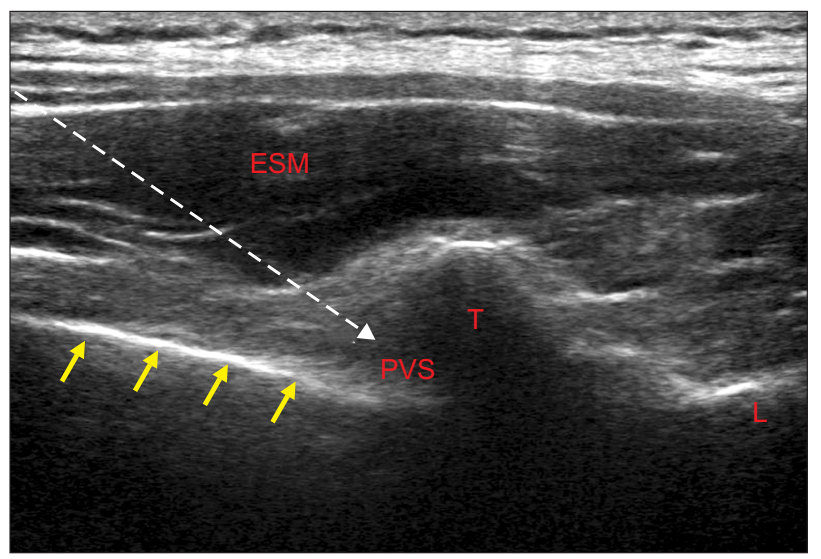

Fig. 1. Ultrasound image of the paravertebral block in transverse approach technique. The ultrasound probe is placed in the transverse axis. The paravertebral space is located beneath the transverse process seen in the image and above the pleura (arrows). The needle is inserted in-plane in the lateral to medial direction. White dashed line with arrow presents the needle trajectory. Arrows: pleura, ESM: erector spinae muscle, L: lamina, PVS: paravertebral space, T: transverse process. 
eter may be inserted if continuous analgesia is required. An average of $10 \mathrm{ml}$ LA will reportedly block approximately five dermatomes [17]. However, some studies indicate that four to six dermatomes are affected following the injection of 20 ml LA $[15,18]$. Therefore, anesthetic spread after a single large volume LA injection is relatively unpredictable, and a multiple injection approach using 3-5 $\mathrm{ml}$ anesthetic for every two adjacent levels of thoracic vertebra may be more practical [19]. The duration of analgesia for a single injection is 3-4 hours for lidocaine and 8-12 hours for ropivacaine or bupivacaine [14].

\section{Parasagittal approach}

For the parasagittal approach, the ultrasound probe is positioned in the vertical plane lateral to the spinous process of the thoracic spine in the paravertebral space. First, the ribs from the corresponding dermatomes and pleura are identified. Next, the ultrasound probe is moved in a medial direction to locate the transverse process. If the probe is moved slightly further in the medial direction, the rib is masked by the transverse process and contour of the transverse process, and the rib appears as a single structure (Fig. 2). The block

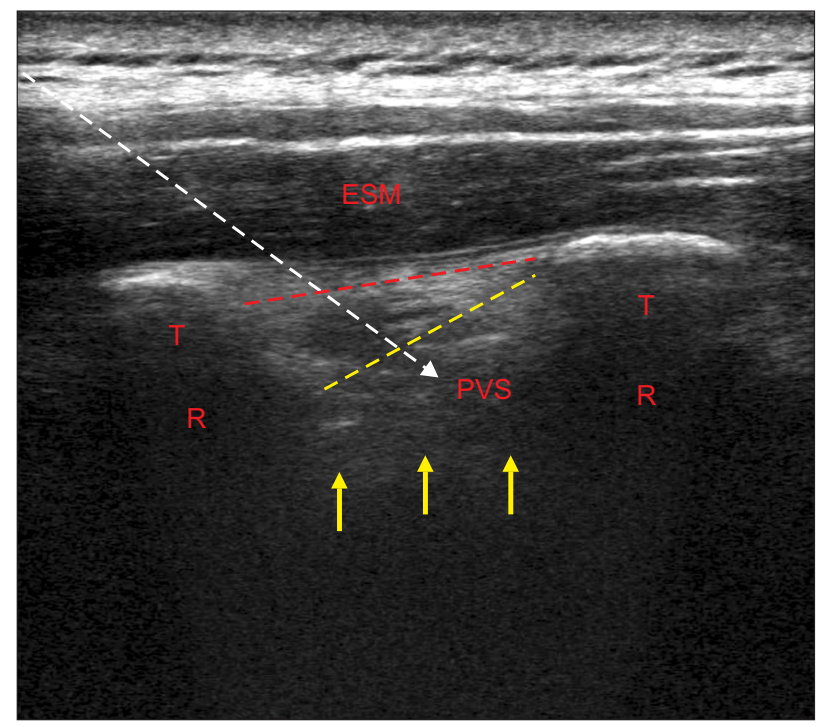

Fig. 2. Ultrasound image of the paravertebral block in parasagittal approach technique. The ultrasound probe is placed in the parasagittal axis. The paravertebral space is located under the superior costotransverse ligament (yellow dashed line) and above the pleura (arrows). The needle is inserted in-plane in the caudal to cephalad direction (white dashed line with arrows). Arrows: pleura, Red dashed line: intertrasverse ligament, Yellow dashed line: superior costotransverse ligament, ESM: erector spinae muscle, PVS: paravertebral space, R: rib, T: transverse process. needle is inserted in a caudal to cephalad trajectory in the plane of this ultrasound view, as shown by the arrow in Fig. 2. The needle may need to be inserted at a steeper angle compared to the transverse approach. When inserted into the paravertebral space, the needle will penetrate the superior costotransverse ligament, which lies between the upper level thoracic transverse process and the lower rib (Fig. 2). A "pop" is heard when this ligament is penetrated and the needle enters the paravertebral space; if a low resistance syringe is being used, a loss of resistance will also be felt. A Tuohy needle may be required for this PVB technique. This is because the needle tip may not always be visible due to the steep angle of approach and it is important to take care not to damage the pleura. Compared to the transverse approach, the parasagittal approach has a lower risk for unintended epidural injection and epidural catheter migration [14].

\section{Evidence and clinical applications}

A thoracic PVB is indicated to provide perioperative pain relief for various abdominal surgeries including renal surgeries, herniorrhaphies, cholecystectomies, and other major abdominal procedures involving the thoracic spinal nerve dermatomes. It is also indicated for pain relief after breast surgeries, thoracic surgeries, and surgeries involving the chest wall. Several studies have compared the efficacy of the thoracic $\mathrm{PVB}$, thoracic epidural analgesia (TEA), and other postthoracotomy pain-management modalities. A systematic review by Joshi et al. [20] demonstrated that the thoracic PVB provided superior pain relief and a reduced requirement for opioids compared to the intercostal nerve block, intrapleural analgesia, and intrathecal opioids. For post-thoracotomy pain, the analgesic efficacy of the thoracic PVB was equal to or greater than that of TEA $[5,21,22]$. Furthermore, the side effect profile of the thoracic PVB was superior to that of TEA and had a higher success rate $[4,21,22]$. A recent Cochrane review meta-analysis by Yeung et al. [4] that included 14 studies with 698 patients concluded that the PVB was as effective as TEA in controlling acute pain and had a reduced risk of complications. However, for the thoracic PVB, the appropriate doses of LA and adjuvants for a single injection and during continuous analgesia remain to be established [14]. The complications that can arise following the thoracic PVB include pneumothorax, hematoma, neurological injury, unintended 
intrathecal or epidural injection, and LA toxicity due to a high infusion dose. In addition, to determine the optimal catheter placement technique for continuous paravertebral analgesia, further studies will be necessary.

\section{INTERCOSTAL NERVE BLOCK}

\section{Clinical background and anatomy}

The intercostal nerve block provides analgesia in the corresponding dermatome, producing band-like anesthesia and analgesia [23]. This block was first described in 1907 by Braun [23]. The intercostal nerves are mixed and contain both motor and sensory fibers, which arise from the ventral rami of the corresponding thoracic spinal nerve roots from $\mathrm{T} 1$ to $\mathrm{T} 11$. The intercostal nerve runs along the inferior aspect of the corresponding rib and into the thoracic pleura and abdominal peritoneum. It provides sensory innervation for the pleura, peritoneum, chest wall, abdominal wall, and groin.

\section{Ultrasound-guided intercostal nerve block techniques}

In most cases, multiple intercostal nerves must be blocked because these do not form a plexus, and each nerve runs along an individual pathway. The number of nerves that need to be blocked will depend on the extent of tissue damage and size of the incision. A dose of 3-5 ml LA for each thoracic nerve level is usually sufficient [24]. To perform the block, the patient should be in the lateral, sitting, or prone position. A high-frequency linear probe is used to generate the ultrasound image. First, the level of the block is marked by counting from the 2 nd or 12 th rib. The tip of the scapula can be a good landmark for the level of the seventh rib [23]. For the initial scan, the ultrasound probe is positioned in the midline along the transverse plane to identify the vertebral level. After the transverse process and rib at the target level have been identified, the probe is moved in a lateral direction following the rib until the rib angle is reached. This can also be determined by palpation if the rib is easy to palpate. Then the target level of the block is marked and the probe is rotated into the parasagittal axis. The ribs are identified as curved structures with an acoustic shadow (Fig. 3). The pleura can be identified as a hyper-echoic line that moves during inspi- ration. The thick erector spinae muscle (ESM) is identified overlying the rib, and three muscle layers (external, internal, and innermost intercostal muscles) are also identified (Fig. 3 ). The intercostal block is normally performed slightly (2 to $3 \mathrm{~cm}$ ) medial to the angle of rib, towards the posterior thorax $[16,25]$. The needle is inserted in-plane in a caudal to cephalad direction and is positioned between the internal intercostal muscle and the innermost intercostal muscle or the pleura at the inferior aspect of the cranial rib (Supplementary Image 2). Approximately 3-5 $\mathrm{ml} \mathrm{LA}$ is injected per level. After the injection of LA, hydrodissection should be performed above the pleura.

\section{Evidence and clinical applications}

The intercostal nerve block is indicated to provide perioperative pain relief for various abdominal and thoracic surgeries. It is also indicated for pain relief after breast surgeries, rib fractures, and chronically painful conditions such as postherpetic neuralgia [26,27]. It can provide sufficient pain relief after thoracotomies, breast surgery, upper abdominal surgeries, and chest traumas such as rib fractures. However, the intercostal nerve block's anatomical coverage overlaps with that of the thoracic PVB, and recent meta-analyses and

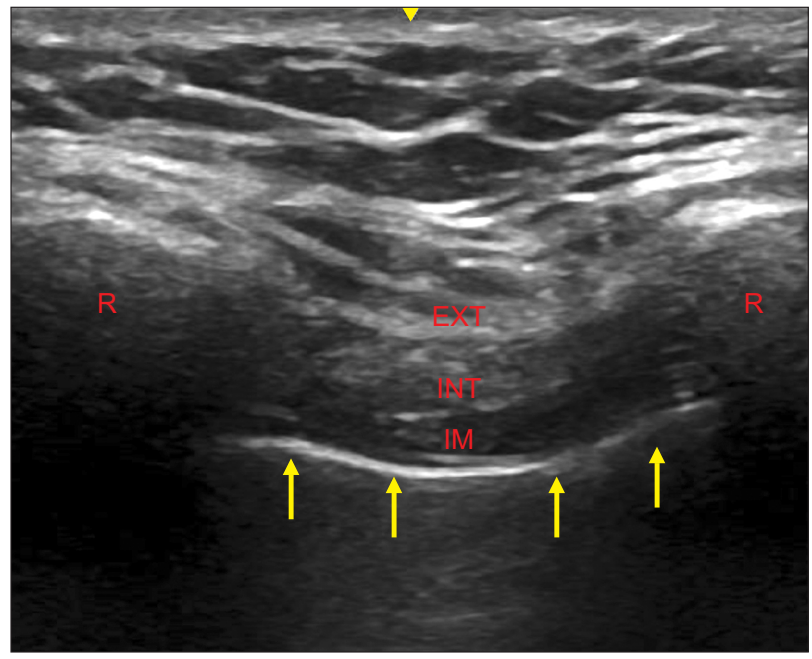

Fig. 3. Ultrasound image of the intercostal block. The ultrasound probe is placed in the parasagittal axis. The needle is inserted in-plane in a caudal to cephalad direction to locate the needle tip between the internal intercostal muscle and the innermost intercostal muscle. Arrows: pleura, EXT: external intercostal muscle, IM: innermost intercostal muscle, INT: internal intercostal muscle, R: rib. 
systematic reviews have suggested that the thoracic PVB is superior for perioperative pain control in patients who have had thoracotomies. Furthermore, whether intercostal nerve blocks are sufficient to manage postoperative pain in breast surgeries is questionable $[20,24]$. The most frequent complication following an intercostal nerve block is a pneumothorax [28]. Other complications include hematoma, hemothorax, lung injury, and the need for intravascular injections due to the proximity of the intercostal vessels and the intercostal nerve.

\section{PECS AND SERRATUS ANTERIOR PLANE BLOCK}

\section{Clinical background and anatomy}

The novel interfascial plane block was first described by Blanco [29] in 2011 and was named the Pecs 1 block. After introducing the Pecs 1 block, Blanco proposed a modification involving the axilla that made the technique suitable for breast surgeries. This was called the Pecs 2 block [30]. Blanco and colleagues [31] also described another variation in the Pecs block to extend the intercostal nerve coverage. This was called the serratus anterior plane block. The Pecs 1 block targets the lateral pectoral nerve and the medial pectoral nerve, which runs between the pectoralis major and pectoralis minor muscles. Therefore, the Pecs 1 block is performed between the fascial plane of the pectoralis major and minor muscles, adjacent to the pectoral branch of the thoracoacromial artery. The Pecs 2 block is an extended version of the Pecs 1 block. First, the Pecs 1 block is performed. Next, LA is injected deep between the serratus anterior and pectoralis minor muscles, at the level of the third and fourth ribs. This blocks the anterior divisions of the lateral rami of the intercostal nerves, long thoracic nerve, and thoracodorsal nerves. The serratus anterior plane block is designed to extend analgesia to the posterior side of the chest wall. It covers the sensory dermatomes from $\mathrm{T} 2$ to $\mathrm{T} 9$. This is a variation in the Pecs 2 block, and the needle is targeted to a more caudal and lateral position, in the mid axillary line at the level of the fifth rib, which is overlain by the serratus anterior and latissimus dorsi muscles. This blocks the lateral cutaneous branches of the intercostal nerve and the long thoracic and thoracodorsal nerves [31,32].

\section{Ultrasound-guided Pecs/serratus anterior block techniques}

\section{Pecs 1 and 2 blocks}

The patient is placed in a supine position and a high-frequency linear probe is used to generate the ultrasound image of the pectoral blocks. The ultrasound probe is positioned in the parasagittal plane in the lower third of the lateral plane, near the clavicle. The first rib visible below the clavicle is the second rib. The superficial pectoralis major muscle and the deeper pectoralis minor muscle are identified, and the needle is inserted in-plane in a cranial to caudal direction (Fig. 4). A total dose of 10-20 $\mathrm{ml} \mathrm{LA}$ is injected between the fascia of the two muscle layers (Pecs 1, Supplementary Image 3 ). To perform the Pecs 2 block, the third and fourth ribs are identified by counting from the second rib in the ultrasound image. After performing the Pecs 1 block, described above, the needle is positioned deeper between the pectoralis minor and serratus anterior muscles (Supplementary Image 4). Another dose of $20 \mathrm{ml} \mathrm{LA}$ is injected between the two muscles.

\section{Serratus anterior block}

The patient is placed in the sitting or lateral position, and a high-frequency linear probe is selected. The ultrasound probe is used to scan beneath the clavicle and the second rib is identified. Next, the probe is moved laterally and caudally

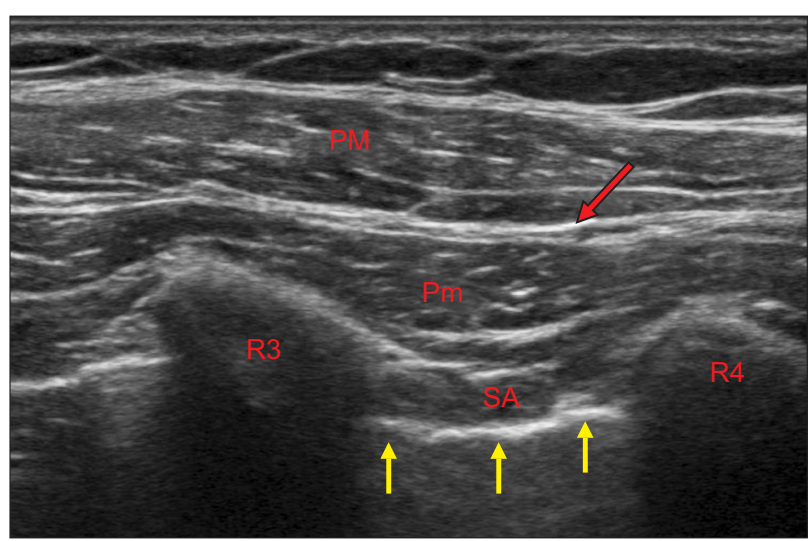

Fig. 4. Ultrasound image of the Pecs 2 block. The ultrasound probe is placed in the parasagittal axis at the level of 3rd and 4th rib. The needle is inserted in-plane in a cranial to caudal direction to locate between 1) the pectoralis major and minor; and 2) pectoralis minor and serratus anterior muscle. Red arrow: thoracodorsal artery, Yellow arrows: pleura, PM: pectoralis major, Pm: pectoralsis minor, R3: third rib, R4: fourth rib, SA: serratus anterior. 
toward the mid-axillary line and the ribs are counted. The fourth and fifth ribs and the serratus anterior and latissimus dorsi muscles are identified in the ultrasound image. A total of $20 \mathrm{ml} \mathrm{LA}$ solution is injected either superficial to the serratus anterior (between the latissimus dorsi and the serratus anterior) or deep between the serratus anterior and external intercostal muscles.

\section{Evidence and clinical applications}

The Pecs blocks and the serratus anterior block were initially developed to provide analgesia after breast surgery. In addition, they are indicated for perioperative pain relief in surgeries involving the anterior chest wall and the axilla (Pecs 2) or the posterolateral chest wall (serratus anterior block). However, these blocks are unable to anesthetize the anterior cutaneous branches of the intercostal nerves; therefore, the medial aspect of the breast including the nipple and areola are not blocked [24]. To additionally block the anterior cutaneous branches, the pectointercostal and transversus thoracic muscle plane blocks have recently been described [32-34]. However, further studies will be required to confirm the extent of LA spread and analgesia. Several studies have recently described the efficacy of pectoral plane blocks. These demonstrate that the Pecs blocks are associated with lower pain scores and opioid sparing effects compared to placebo $[35,36]$. Comparing the Pecs blocks with single thoracic paravertebral injections has produced conflicting efficacy results $[37,38]$. Potential complications following these blocks include pneumothorax and injury to the thoracodorsal artery. However, because these techniques are relatively novel, few data regarding complications have been collected [39].

\section{TAP BLOCK}

\section{Clinical background and anatomy}

The TAP block was introduced in 2001 as a landmarkguided technique [40]. Because the widespread introduction of ultrasound guidance for peripheral nerve blocks, interest has developed in using the TAP block for perioperative analgesia in various types of surgery involving the abdomen. The anterior rami of the thoracolumbar nerves from $\mathrm{T} 6$ to L1 innervate the segmental cutaneous anterior abdominal wall; this includes the intercostal (T6 to T11), subcostal (T12), ilioinguinal and iliohypogastric (L1) nerves [41]. The branches inter-communicate and form the upper and lower TAP plexuses and the rectus sheath plexus. These segmental nerve branches converge just above the transversus abdominis muscle or between the transversus and the internal oblique abdominis muscles $[41,42]$. Therefore, LA applied in this plane will block these nerve plexus branches and provide analgesia to the anterolateral abdominal wall $[1,41,42]$. There are several TAP block approaches, which differ depending on the point of injection. These include the subcostal TAP (T6 to T9), the oblique subcostal TAP (T6 to L1; which corresponds to the upper approach TAP), the lateral or mid-axillary approach TAP (T10 to T12), and the posterior approach TAP (T9 to T12) [42]. The dual TAP combines the subcostal/oblique subcostal (upper) TAP and the lateral/posterior TAP techniques. The dual TAP block can provide analgesia to both the supra and infraumbilical hemi-abdomen. If analgesia of the entire anterolateral abdominal wall is required, then a bilateral dual TAP block called a "four quadrant" TAP block can

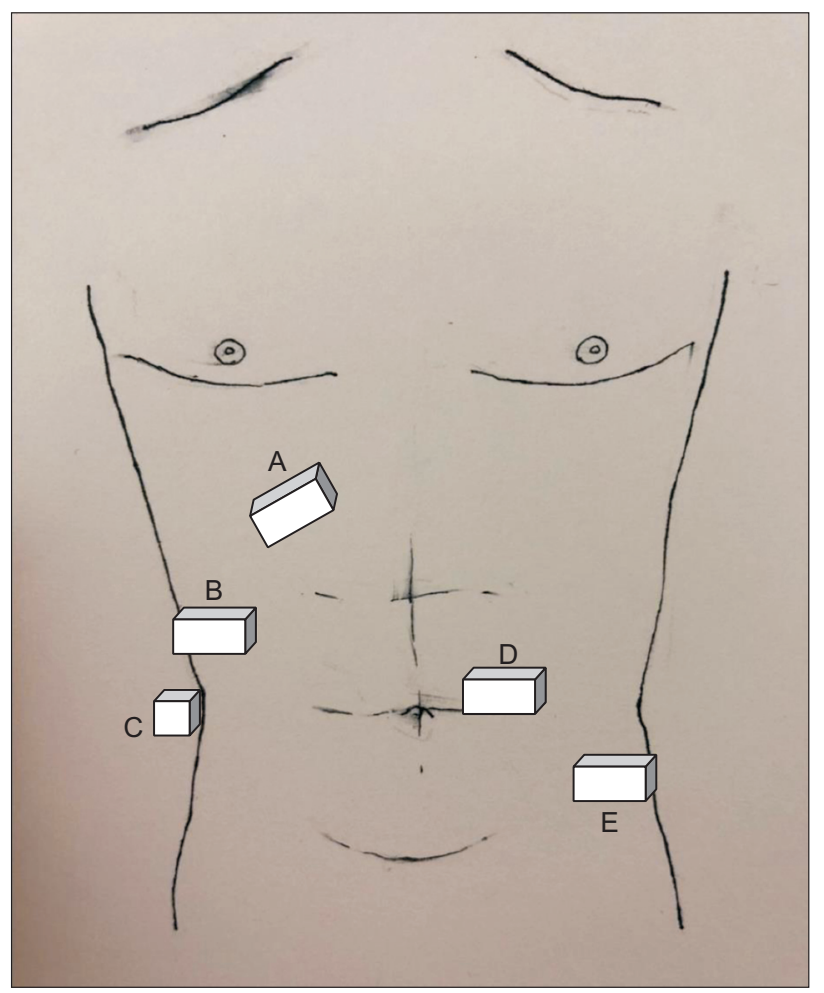

Fig. 5. Ultrasound probe positions for the transversus abdominis plane (TAP) block, rectus sheath block and ilioinguinal nerve block. (A) subcostal TAP block, (B) lateral TAP block, (C) posterior TAP block, (D) rectus sheath block, $(E)$ ilioinguinal nerve block. 
be performed $[43,44]$. The position of the ultrasound probe will depend on the type of TAP block, as shown in Fig. 5.

\section{Ultrasound-guided TAP block techniques}

The patient is placed in a supine position for the TAP block. When performing the lateral or posterior TAP block, the patient may be placed in a lateral position. A high-frequency linear ultrasound probe is used for scanning.

\section{Subcostal TAP block}

The ultrasound probe is held at an oblique angle in the transverse position beneath the xiphoid process and is moved in a lateral direction, parallel to the costal margin (Fig. 5). The rectus abdominis is shown in Fig. 6, and the transversus abdominis is below the rectus abdominis. A total of $15-20 \mathrm{ml} \mathrm{LA}$ is injected between the transversus abdominis and the rectus abdominis. If the transversus abdominis terminates lateral to the rectus abdominis, the LA can be deposited between the transversus and internal oblique muscles, just lateral to the linea semilunaris. The subcostal TAP block provides analgesia to the anterior abdomen above the umbilicus.

\section{Lateral TAP block}

The probe is moved in a lateral direction along the costal margin until the mid-axillary line is almost reached. Then the probe is held in the transverse position between the costal margin and iliac crest (Fig. 5). The three layers of abdomi-

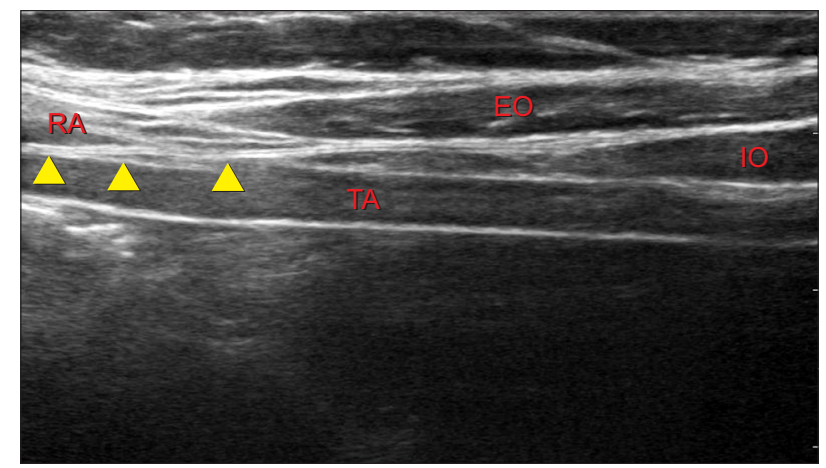

Fig. 6. Ultrasound image of the subcostal transversus abdominis plane block. The needle is positioned in-plane between the transversus abdominis muscle and the rectus abdominis muscle (arrow heads). Arrow heads: target plane of local anesthetic deposit, EO: external oblique, IO: internal oblique, RA: rectus abdominis, TA: transversus abdominis muscle. nal muscles (internal and external oblique abdominis and transversus abdominis muscles) will appear (Fig. 7). A total of 15-20 $\mathrm{ml} \mathrm{LA}$ is injected between the transversus abdominis and the internal oblique abdominis muscle layers (Supplementary Image 5). The lateral TAP block provides analgesia to the anterior abdomen below the umbilicus.

\section{Posterior TAP block}

From its starting position for the lateral TAP block, the ultrasound probe is moved in a lateral and posterior direction until an ultrasound image is generated that shows the point where the three layers of abdominal muscles end and the transversus abdominis muscle becomes an aponeurosis (Fig. 8). The QL muscle can be seen below the aponeurosis, and latissimus dorsi muscle is visible lateral to the internal and external oblique abdominis muscles. A total of 15-20 ml LA is injected between the transversus abdominis and the internal oblique abdominis muscle layers or above the aponeurosis of the transversus abdominis (Supplementary Image 6). The posterior TAP block provides extended analgesia compared to the lateral TAP block, additionally anesthetizing the lateral and some of the posterior abdominal wall.

\section{Evidence and clinical applications}

Following the more widespread application of ultrasound for assisting nerve block techniques, the TAP block has been

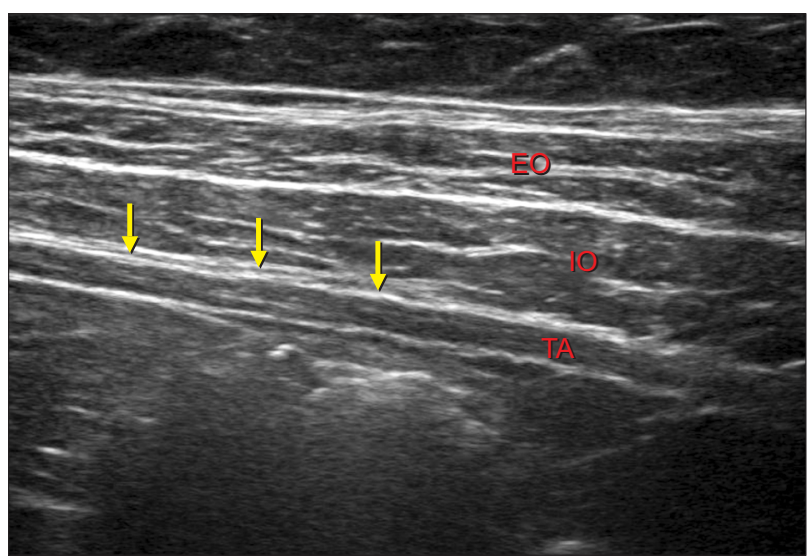

Fig. 7. Ultrasound image of the lateral transversus abdominis plane block. The needle is positioned in-plane between the transversus abdominis muscle and the internal oblique abdominis muscle (arrows). Arrows: target plane of local anesthetic deposit, EO: external oblique, IO: internal oblique, TA: transversus abdominis muscle. 


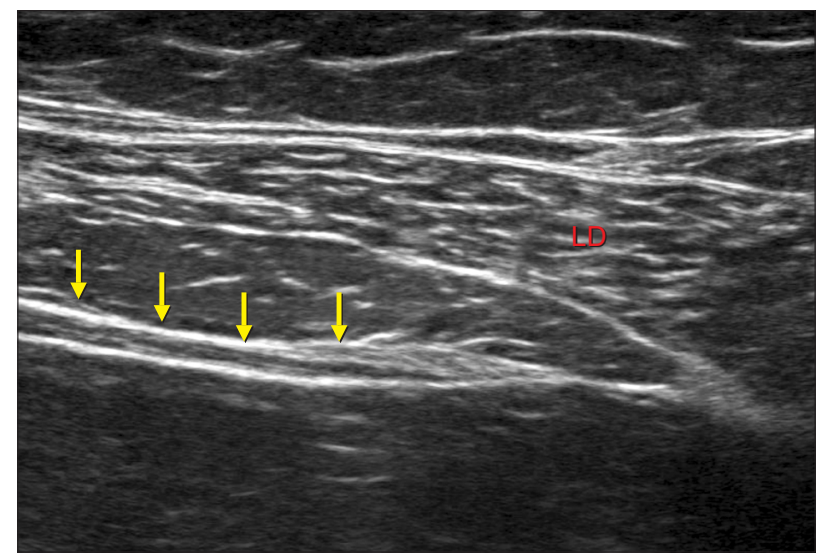

Fig. 8. Ultrasound image of the posterior transversus abdominis plane block. The needle is positioned in-plane above the aponeurosis of the transversus abdominis muscle (arrows). Arrows: target plane of local anesthetic deposit, LD: latissimus dorsi muscle.

used for perioperative analgesia in various types of abdominal surgeries and extensive studies have demonstrated its efficacy. Although the TAP block only alleviates somatic pain, it provides effective analgesia following abdominal surgeries when combined with multimodal analgesic techniques [45]. Compared to placebo, the TAP block reduces pain scores and opioid consumption following various lower-abdomina surgeries such as anterior iliac crest bone harvesting [46], hysterectomy [47], prostatectomy [48], cesarean section [49], and major surgery of the upper abdomen $[3,50]$. The fourquadrant TAP block with a continuous posterior TAP catheter showed comparable levels of analgesia and non-inferior postoperative pain scores and rescue analgesic consumption levels compared to continuous TEA [2]. Despite a relatively large number of studies and evidence of efficacy, selecting the best approach for each specific surgical procedure remains challenging [51]. For example, a posterior TAP block has been recommended for pain control following incisions under the umbilicus, and a subcostal or oblique subcostal TAP block may be most suitable for incisions involving the supraumbilical abdominal wall. Reports of complications following ultrasound-guided TAP blocks have been rare, and as such, it is considered a relatively simple and safe procedure. Potential complications include organ damage and local systemic toxicity due to the relatively large injection volume used in this procedure $[52,53]$.

\section{RECTUS SHEATH BLOCK}

\section{Clinical background and anatomy}

The rectus sheath block was first described in 1899 to relax the muscles of the abdominal wall. In 1996, it was suggested that this old technique might provide analgesia following umbilical hernia repairs in children [54]. After the more widespread application of ultrasound for assisting peripheral nerve block techniques in the mid-2000s, the rectus sheath block was applied to provide perioperative analgesia in operations involving periumbilical and midline incisions of the abdomen [55-58]. Depositing LA in the rectus sheath beneath the rectus abdominis muscle blocks the terminal anterior cutaneous branches of the intercostal nerves from T9 to T11, which transfer sensory information from the periumbilical skin. The rectus sheath block is usually performed bilaterally as it is widely used for analgesia in surgeries involving midline incisions.

\section{Ultrasound-guided rectus sheath block techniques}

The patient is placed in a supine position and the ultrasound probe is held in the transverse position over the rectus abdominis muscle (Fig. 9). A high-frequency linear probe is used to perform the rectus sheath block. The needle is inserted between the rectus abdominis muscle and the posterior rectus sheath, and 15-20 ml LA is injected. Hydrodissection is used to separate the rectus abdominis muscle and the posterior rectus sheath (Supplementary Image 7).

\section{Evidence and clinical applications}

Compared to placebo or local wound infiltration analgesia, the rectus sheath block results in lower pain scores with opioid-sparing effects in both children and adults receiving umbilical hernia repair $[56,57,59]$. Study reports have also demonstrated that this block provides effective pain control in surgeries involving midline incisions $[60,61]$. The rectus sheath block can be used in combination with the TAP block for pain relief over a wider area in surgeries involving the anterior abdominal wall $[57,62]$. Potential complications following the rectus sheath block include peritoneal or bowel 


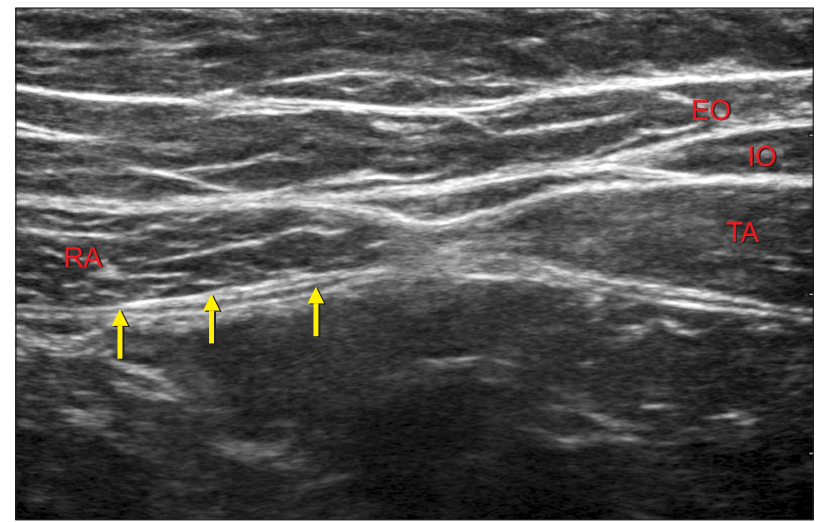

Fig. 9. Ultrasound image of the rectus sheath plane block. The needle is positioned in-plane under the rectus abdominis muscle and the posterior rectus sheath (arrows). Arrows: target plane of local anesthetic deposit, EO: external oblique, IO: internal oblique, RA: rectus abdominis, TA: transversus abdominis muscle.

perforation, hematoma, and local systemic toxicity.

\section{ILIOINGUINAL AND ILIOHYPOGASTRIC NERVE BLOCKS}

\section{Clinical background and anatomy}

These blocks were performed using landmarks since the 1980s. However, landmark-based ilioinguinal and iliohypogastric nerve blocks had low success rates, and much of the anesthetic was administered intramuscularly $[63,64]$. The introduction of ultrasound for assisting nerve blocks has increased success rates and improved outcomes. The ilioinguinal and iliohypogastric nerves separate from the L1 spinal nerve, emerge from the outer border of the upper part of the psoas major muscle, and cross the QL muscle. They penetrate the transversus abdominis muscle near the anterior iliac crest and communicate with each other between the transversus abdominis and the internal oblique muscle. Blocking the ilioinguinal and iliohypogastric nerves by infiltrating LA anesthetizes the inguinal wall, upper thigh, and suprapubic areas. These two techniques are similar to the TAP block. Blocking the ilioinguinal and iliohypogastric nerves separately is challenging [65].

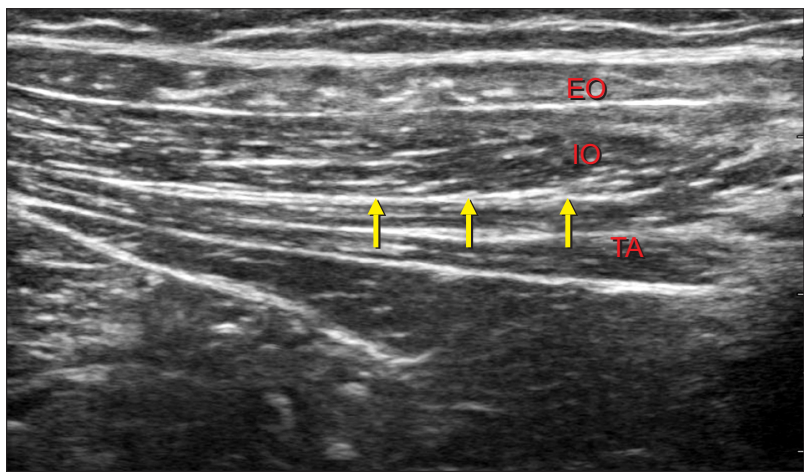

Fig. 10. Ultrasound image of the ilioinguinal and iliohypogastric nerve blocks. The needle is positioned in-plane in the lateral to medial direction between the transversus abdominis muscle and the internal oblique abdominis muscle (arrows). The bony shadows in the right lower corner is the anterior superior iliac spine. Arrows: target plane of local anesthetic deposit, EO: external oblique, IO: internal oblique, TA: transversus abdominis muscle.

\section{Ultrasound-guided iliohypogastric nerve block techniques}

The patient is placed in a supine position. A high-frequency linear ultrasound probe is used for scans. The ultrasound probe is held at an oblique angle and moved from the anterior superior iliac spine (ASIS) to the umbilicus. The three abdominal muscle layers are identified, and $10-20 \mathrm{ml}$ LA is deposited in the fascia layer between the internal oblique and transversus abdominis muscles (Fig. 10). It is important to perform this block close and proximal to the ASIS, at a point before the nerve branches.

\section{Evidence and clinical applications}

The ilioinguinal and iliohypogastric nerve blocks are indicated for surgeries in the inguinal and suprapubic area. There is good evidence that these blocks provide effective perioperative pain control for inguinal hernia repairs in children and adults [66-68]. The bilateral block reduces opioid consumption after cesarean sections and other gynecological surgeries performed on the lower abdomen $[69,70]$. No serious complications have been reported following iliohypogastric nerve blocks, but there is the possibility of intraperitoneal injection and bowel injuries. 


\section{QL BLOCK}

\section{Clinical background and anatomy}

The QL block was first described by Blanco in 2007 as an extension of the posterior TAP block [51]. The posterior TAP block, performed at the intersection between the QL muscle and the abdominal wall muscles just superficial to the transversus abdominis aponeurosis, results in the spread of LA around the QL and psoas muscles. Furthermore, magnetic resonance imaging has demonstrated that some paravertebral/epidural spread of LA occurs between T5 and T10 [71]. However, the QL block technique has been redefined with the LA deposited under the transversus aponeurosis and along the lateral aspect of the QL muscle [72]. Four types of QL block have been described according to the injection site relative to the QL muscle. These include the anterior (transmuscular), lateral (type 1), posterior (type 2), and intramuscular QL block techniques [72]. The thoracolumbar fascia is the fascial layer that encases the back muscles and consists of anterior, middle, and posterior layers. The anterior layer is located anterior to the QL muscle, the middle layer lies between the QL and the ESMs, and the posterior layer is located posterior to the QL muscle. As a result, LA deposited in the posterior and middle thoracolumbar fascial layer (lateral and posterior QL blocks) provides more extensive anesthesia and analgesia of the abdominal wall compared to the posterior TAP block. LA spread anterior to the QL muscle (anterior QL block) should further block the branches of the lumbar plexus, providing an additional analgesic effect to the lower extremities [73,74].

\section{Ultrasound-guided QL block techniques}

For the posterior and lateral QL blocks, a high-frequency linear ultrasound probe may be suitable, but for obese patients and for the anterior QL block, a low frequency curved probe should be used. The ultrasound scanning technique is similar to that previously described for the posterior TAP block.

\section{Anterior QL block}

The patient is placed in a lateral or prone position. The probe is held in the transverse position above the iliac crest, and an ultrasound image of the point where the three layers of abdominal muscles terminate and the transversus abdominis muscle becomes an aponeurosis is identified. The QL and latissimus dorsi muscles are identified medial/ posterior to the transversus aponeurosis. The probe is moved slightly toward the spinal midline until the psoas major and ESMs become visible (Fig. 11). The needle tip is targeted to the fascial plane, between the psoas and QL muscles, and approximately $20 \mathrm{ml} \mathrm{LA}$ is injected.

\section{Posterior QL block}

The patient is placed in a lateral or supine position with a pillow under the back. The ultrasound scanning technique is identical to that described for the anterior QL block (Fig. 11). After the QL muscle is identified, $20 \mathrm{ml}$ LA is injected at the fascial layer along the posterior aspect of the QL muscle or between the QL and ESMs.

\section{Lateral QL block}

The patient is placed in a supine position. The ultrasound scanning technique is identical to that described for the posterior TAP block. LA is injected under the transversus apo-

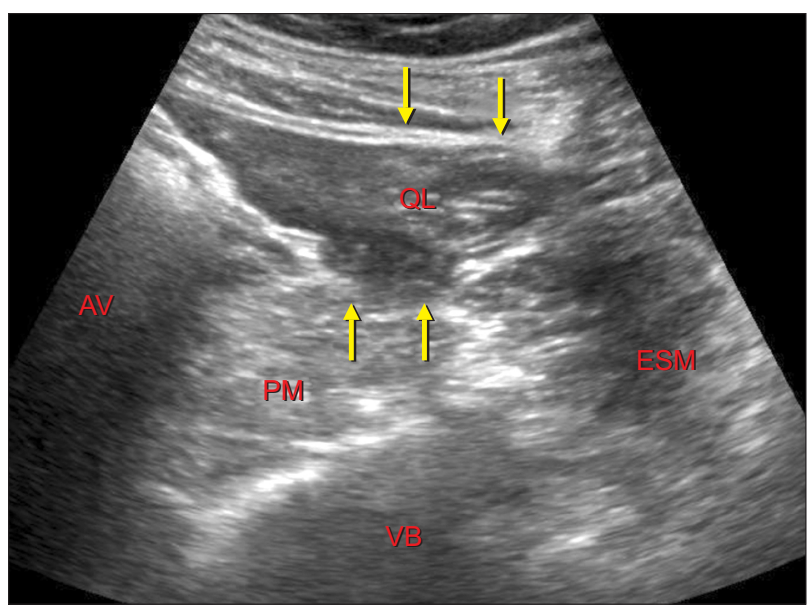

Fig. 11. Ultrasound image of the quadratus lumborum (QL) block. The "Shamrock" image is obtained. 1) Posterior QL block: the needle is positioned in the fascial layer posterior to the QL muscle (upper arrows), 2) anterior QL block: the needle is positioned in between the QL muscle and the psoas major muscle (lower arrows). Upper (down facing) arrows: target plane of local anesthetic deposit for the posterior QL block, Lower (upper facing) arrows: target plane of local anesthetic deposit for the anterior QL block, AV: abdominal viscera, ESM: erector spinae muscle, PM: psoas major muscle, QL: quadratus lumborum muscle, VB: vertebral body. 
neurosis at the anterolateral border of the QL muscle (note that LA is injected superficial to the transversus aponeurosis for the posterior TAP block).

\section{Evidence and clinical applications}

The QL block is capable of providing analgesia in most cases of abdominal surgery. The lateral and posterior QL block provides more extensive analgesia compared to the posterior TAP block and can generate analgesia from T6-7 to L1 $[75,76]$. Furthermore, the anterior QL block can provide analgesia to the lower extremities, which is useful for surgeries involving the hip and thigh. The anterior QL block can also provide analgesia from T10 to L3 [76]. A study by Blanco et al. [77,78] demonstrated that the QL block resulted in significantly less postoperative pain and opioid consumption compared to placebo in cesarean section patients. The QL block was also superior to the posterior TAP block and resulted in less postoperative opioid consumption. However, further randomized controlled studies will be necessary to determine the optimal applications of this technique and identify relevant differences between the lateral and posterior QL blocks and the posterior TAP block. Potential complications include penetration of the peritoneum and associated organ injuries, kidney damage, and local systemic toxicity due to the relatively large volume of LA required for bilateral blocks.

\section{ESP BLOCK}

\section{Clinical background and anatomy}

One truncal block that has attracted a lot of recent interest is the ESP block. The ESP block was first described by Forero et al. [79], who tried to produce analgesia in patients with thoracic neuropathic pain. More than 40 cases that involve this novel blocking technique have been published since. These numerous case reports suggest that the many indications for the ESP block include ventral hernias, rib fractures, thoracotomies, visceral abdominal analgesia, breast surgeries, chronic shoulder pain, inguinal hernia in infants, and cesarean sections. However, as with most other truncal blocks, a deeper understanding of the anatomical basis of the ESP block is required. In a radiological imaging study that included fresh cadavers and living patients, following the ESP block applied at the fifth thoracic vertebral level, the injectate spread widely in the cephalad and caudal directions along the fascial sheath surrounding the ESM [80]. This suggested that the site of action is the dorsal and ventral rami of the thoracic spinal nerves. The spread of the drug, anterior and inferior to the ESM fascial sheath, resembles the pattern of drug infiltration during the PVB. In addition, the spread of the drug outward and posterior to the ESM fascial sheath resembles the pattern of drug infiltration during the QL or the posterior TAP block. The advantage of the ESP block is that it requires less technical expertise because it is similar to other truncal blocks and technically easier to perform compared to neuraxial, nerve plexus, and targeted nerve blocks. In addition, it is likely to have fewer serious side effects (e.g., spinal cord damage, nerve trauma, pneumothorax). Although it is possible to administer a continuous block by inserting a catheter, there has been little research on whether the ESP block can be effective as a single regional technique.

\section{Ultrasound-guided ESP block techniques}

The patient is placed in a sitting position; a lateral position may also be suitable for this block. A high-frequency linear

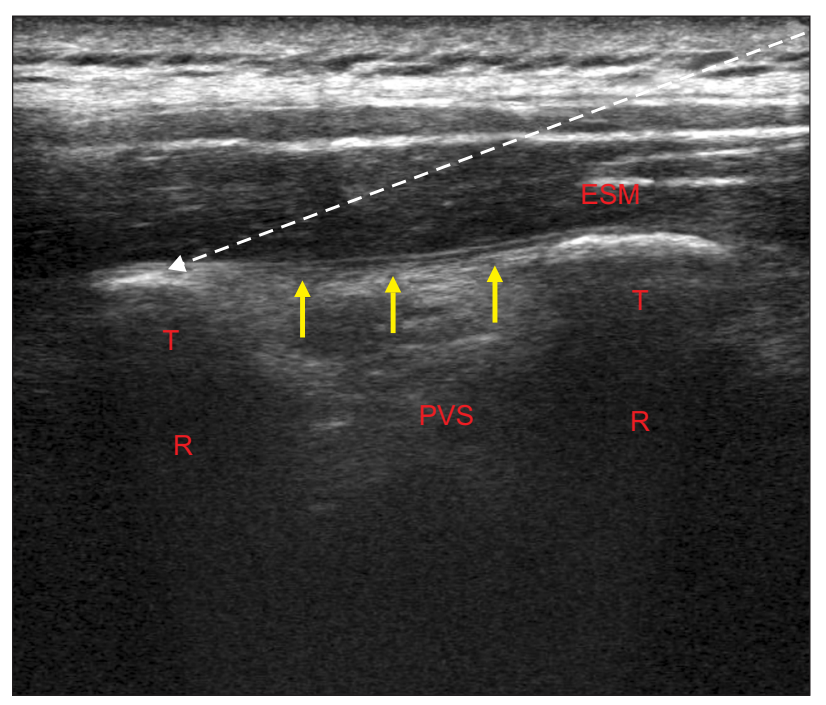

Fig. 12. Ultrasound image of the erector spinae plane block. The ultrasound probe is placed in the parasagittal axis. The needle is inserted in-plane in a cranial to caudal direction to locate the needle tip between the transverse process and the erector spinae muscle (white dashed line with arrows). White dashed line with arrow presents the needle trajectory. Arrows: target plane of local anesthetic deposit, ESM: erector spinae muscle, PVS: paravertebral space, R: rib, T: transverse process. 
probe is used. The probe is positioned in the parasagittal plane and the scanning technique is similar to that described for the thoracic PVB. The contour of the target transverse process (T5 for thoracic and upper abdominal procedures; $\mathrm{T} 7$ for lower abdominal procedures) will be visible in the ultrasound image (Fig. 12). The needle is inserted in-plane in a cranial to caudal direction until it contacts the transverse process. Injecting $<1 \mathrm{ml}$ fluid should separate the ESM from the transverse process and confirm the correct location of the block needle. A total of $20-30 \mathrm{ml}$ LA is injected in the fascial plane beneath the ESM.

\section{Evidence and clinical applications}

Numerous applications of this block technique have been described since it was introduced. However, these are case reports and case series, and prospective or retrospective studies assessing the efficacy of the technique have yet to be published. The authors who first described the ESP block believe that as an analgesic block, it is similar to the PVB. However, it is associated with a much lower risk of side effects and can be safely performed in higher risk patients, such as patients with coagulation disorders. These features make the ESP block a simple and safe technique that is suitable for treating acute chest and abdominal pain following surgery. Prospective studies will be required to confirm its efficacy, safety, and clinical applications.

\section{CONCLUSIONS}

Advances in ultrasound technology and their widespread applications in clinical anesthesiology have led to renewed interest in the use of ultrasound-guided truncal blocks. These blocks are relatively easy to perform and have less serious side effects than central neuraxial nerve block techniques. As a result, these techniques are being used more frequently to produce perioperative analgesia for surgeries involving the thorax and abdomen. Many randomized controlled studies have shown that correctly applied ultrasound-guided truncal blocks provide non-inferior analgesia and superior safety profiles compared to central neuraxial analgesic techniques $[2-5,21]$. However, to apply each block correctly and to reduce the likelihood of related side effects and complications, the practitioner must have a thorough understanding of the anatomical region, optimal block positioning, and device selection and management. Furthermore, ultrasound-guided truncal blocks are part of a new and constantly changing field of anesthesiology; novel techniques have recently been described, including the ESP block and the superior gluteal nerve block $[79,81]$. Therefore, additional efforts will be required to provide evidence regarding the efficacy and safety of these new blocking techniques. Only then can the application of ultrasound-guided truncal blocks be supported by both research and education.

\section{SUPPLEMENTARY MATERIALS}

Supplementary data is available at https://doi.org/10.17085/ apm.2018.13.2.128.

\section{REFERENCES}

1. Finnerty O, Carney J, McDonnell JG. Trunk blocks for abdominal surgery. Anaesthesia 2010; 65 Suppl 1: 76-83.

2. Niraj G, Kelkar A, Hart E, Horst C, Malik D, Yeow C, et al. Comparison of analgesic efficacy of four-quadrant transversus abdominis plane (TAP) block and continuous posterior TAP analgesia with epidural analgesia in patients undergoing laparoscopic colorectal surgery: an open-label, randomised, noninferiority trial. Anaesthesia 2014; 69: 348-55.

3. Niraj G, Kelkar A, Jeyapalan I, Graff-Baker P, Williams O, Darbar A, et al. Comparison of analgesic efficacy of subcostal transversus abdominis plane blocks with epidural analgesia following upper abdominal surgery. Anaesthesia 2011; 66: 465-71.

4. Yeung JH, Gates S, Naidu BV, Wilson MJ, Gao Smith F. Paravertebral block versus thoracic epidural for patients undergoing thoracotomy. Cochrane Database Syst Rev 2016; 2: CD009121.

5. Ding X, Jin S, Niu X, Ren H, Fu S, Li Q. A comparison of the analgesia efficacy and side effects of paravertebral compared with epidural blockade for thoracotomy: an updated meta-analysis. PLoS One 2014; 9: e96233.

6. Wilson SH, Wolf BJ, Algendy AA, Sealy C, Demos HA, McSwain JR. Comparison of lumbar epidurals and lumbar plexus nerve blocks for analgesia following primary total hip arthroplasty: a retrospective analysis. J Arthroplasty 2017; 32: 635-40.

7. Karmakar MK. Thoracic paravertebral block. Anesthesiology 2001; 95: 771-80.

8. Luyet C, Eichenberger U, Greif R, Vogt A, Szücs Farkas Z, Moriggl B. Ultrasound-guided paravertebral puncture and placement of catheters in human cadavers: an imaging study. Br J Anaesth 
2009; 102: 534-9.

9. Hara K, Sakura S, Nomura T, Saito Y. Ultrasound-guided thoracic paravertebral block in breast surgery. Anaesthesia 2009; 64: 2235.

10. Shibata Y, Nishiwaki K. Ultrasound-guided intercostal approach to thoracic paravertebral block. Anesth Analg 2009; 109: 996-7.

11. Ben-Ari A, Moreno M, Chelly JE, Bigeleisen PE. Ultrasoundguided paravertebral block using an intercostal approach. Anesth Analg 2009; 109: 1691-4.

12. Eason MJ, Wyatt R. Paravertebral thoracic block-a reappraisal. Anaesthesia 1979; 34: 638-42.

13. Bouman EAC, Sieben JM, Balthasar AJR, Joosten EA, Gramke $\mathrm{HF}$, van Kleef M, et al. Boundaries of the thoracic paravertebral space: potential risks and benefits of the thoracic paravertebral block from an anatomical perspective. Surg Radiol Anat 2017; 39: 1117-25.

14. D'Ercole F, Arora H, Kumar PA. Paravertebral block for thoracic surgery. J Cardiothorac Vasc Anesth 2018; 32: 915-27.

15. Cowie B, McGlade D, Ivanusic J, Barrington MJ. Ultrasoundguided thoracic paravertebral blockade: a cadaveric study. Anesth Analg 2010; 110: 1735-9.

16. Krediet AC, Moayeri N, van Geffen GJ, Bruhn J, Renes S, Bigeleisen PE, et al. Different approaches to ultrasound-guided thoracic paravertebral block: an illustrated review. Anesthesiology 2015; 123: $459-74$.

17. Saito T, Den S, Cheema SP, Tanuma K, Carney E, Carlsson C, et al. A single-injection, multi-segmental paravertebral block-extension of somatosensory and sympathetic block in volunteers. Acta Anaesthesiol Scand 2001; 45: 30-3.

18. Renes SH, Bruhn J, Gielen MJ, Scheffer GJ, van Geffen GJ. Inplane ultrasound-guided thoracic paravertebral block: a preliminary report of 36 cases with radiologic confirmation of catheter position. Reg Anesth Pain Med 2010; 35: 212-6.

19. Kotzé A, Scally A, Howell S. Efficacy and safety of different techniques of paravertebral block for analgesia after thoracotomy: a systematic review and metaregression. Br J Anaesth 2009; 103: 626-36.

20. Joshi GP, Bonnet F, Shah R, Wilkinson RC, Camu F, Fischer B, et al. A systematic review of randomized trials evaluating regional techniques for postthoracotomy analgesia. Anesth Analg 2008; 107: 1026-40.

21. Davies RG, Myles PS, Graham JM. A comparison of the analgesic efficacy and side-effects of paravertebral vs epidural blockade for thoracotomy--a systematic review and meta-analysis of randomized trials. Br J Anaesth 2006; 96: 418-26.

22. Scarci M, Joshi A, Attia R. In patients undergoing thoracic surgery is paravertebral block as effective as epidural analgesia for pain management? Interact Cardiovasc Thorac Surg 2010; 10: 92-6.

23. Bhatia A, Gofeld M, Ganapathy S, Hanlon J, Johnson M. Comparison of anatomic landmarks and ultrasound guidance for intercostal nerve injections in cadavers. Reg Anesth Pain Med 2013; 38: 503-7.

24. Woodworth GE, Ivie RMJ, Nelson SM, Walker CM, Maniker RB. Perioperative breast analgesia: a qualitative review of anatomy and regional techniques. Reg Anesth Pain Med 2017; 42: 609-31.

25. Chakraborty A, Khemka R, Datta T. Ultrasound-guided truncal blocks: a new frontier in regional anaesthesia. Indian J Anaesth 2016; 60: 703-11.

26. Byas-Smith MG, Gulati A. Ultrasound-guided intercostal nerve cryoablation. Anesth Analg 2006; 103: 1033-5.

27. Ozkan D, Akkaya T, Karakoyunlu N, Arik E, Ergil J, Koc Z, et al. Effect of ultrasound-guided intercostal nerve block on postoperative pain after percutaneous nephrolithotomy: prospective randomized controlled study. Anaesthesist 2013; 62: 988-94.

28. Shanti CM, Carlin AM, Tyburski JG. Incidence of pneumothorax from intercostal nerve block for analgesia in rib fractures. J Trauma 2001; 51: 536-9.

29. Blanco R. The 'pecs block': a novel technique for providing analgesia after breast surgery. Anaesthesia 2011; 66: 847-8.

30. Blanco R, Fajardo M, Parras Maldonado T. Ultrasound description of Pecs II (modified Pecs I): a novel approach to breast surgery. Rev Esp Anestesiol Reanim 2012; 59: 470-5.

31. Blanco R, Parras T, McDonnell JG, Prats-Galino A. Serratus plane block: a novel ultrasound-guided thoracic wall nerve block. Anaesthesia 2013; 68: 1107-13.

32. de la Torre PA, Garcia PD, Alvarez SL, Miguel FJ, Perez MF. A novel ultrasound-guided block: a promising alternative for breast analgesia. Aesthet Surg J 2014; 34: 198-200.

33. Ueshima H, Otake H. Addition of transversus thoracic muscle plane block to pectoral nerves block provides more effective perioperative pain relief than pectoral nerves block alone for breast cancer surgery. Br J Anaesth 2017; 118: 439-43.

34. Ueshima H, Kitamura A. Blocking of multiple anterior branches of intercostal nerves (Th2-6) using a transversus thoracic muscle plane block. Reg Anesth Pain Med 2015; 40: 388.

35. Kamiya Y, Hasegawa M, Yoshida T, Takamatsu M, Koyama Y. Impact of pectoral nerve block on postoperative pain and quality of recovery in patients undergoing breast cancer surgery: a randomised controlled trial. Eur J Anaesthesiol 2018; 35: 215-23.

36. Bashandy GM, Abbas DN. Pectoral nerves I and II blocks in multimodal analgesia for breast cancer surgery: a randomized clinical trial. Reg Anesth Pain Med 2015; 40: 68-74.

37. Kulhari S, Bharti N, Bala I, Arora S, Singh G. Efficacy of pectoral 
nerve block versus thoracic paravertebral block for postoperative analgesia after radical mastectomy: a randomized controlled trial. Br J Anaesth 2016; 117: 382-6.

38. Syal K, Chandel A. Comparison of the post-operative analgesic effect of paravertebral block, pectoral nerve block and local infiltration in patients undergoing modified radical mastectomy: a randomised double-blind trial. Indian J Anaesth 2017; 61: 643-8.

39. Ueshima H, Otake H. Ultrasound-guided pectoral nerves (PECS) block: complications observed in 498 consecutive cases. J Clin Anesth 2017; 42: 46.

40. Rafi AN. Abdominal field block: a new approach via the lumbar triangle. Anaesthesia 2001; 56: 1024-6.

41. McDonnell JG, O'Donnell BD, Farrell T, Gough N, Tuite D, Power $\mathrm{C}$, et al. Transversus abdominis plane block: a cadaveric and radiological evaluation. Reg Anesth Pain Med 2007; 32: 399-404.

42. Tsai HC, Yoshida T, Chuang TY, Yang SF, Chang CC, Yao HY, et al. Transversus abdominis plane block: an updated review of anatomy and techniques. Biomed Res Int 2017; 2017: 8284363.

43. Børglum J, Maschmann C, Belhage B, Jensen K. Ultrasoundguided bilateral dual transversus abdominis plane block: a new four-point approach. Acta Anaesthesiol Scand 2011; 55: 658-63.

44. Børglum J, Jensen K, Christensen AF, Hoegberg LC, Johansen SS, Lönnqvist PA, et al. Distribution patterns, dermatomal anesthesia, and ropivacaine serum concentrations after bilateral dual transversus abdominis plane block. Reg Anesth Pain Med 2012; 37: 294-301.

45. Abdallah FW, Laffey JG, Halpern SH, Brull R. Duration of analgesic effectiveness after the posterior and lateral transversus abdominis plane block techniques for transverse lower abdominal incisions: a meta-analysis. Br J Anaesth 2013; 111: 721-35.

46. Chiono J, Bernard N, Bringuier S, Biboulet P, Choquet O, Morau $\mathrm{D}$, et al. The ultrasound-guided transversus abdominis plane block for anterior iliac crest bone graft postoperative pain relief: a prospective descriptive study. Reg Anesth Pain Med 2010; 35: 520-4.

47. Carney J, McDonnell JG, Ochana A, Bhinder R, Laffey JG. The transversus abdominis plane block provides effective postoperative analgesia in patients undergoing total abdominal hysterectomy. Anesth Analg 2008; 107: 2056-60.

48. Elkassabany N, Ahmed M, Malkowicz SB, Heitjan DF, Isserman JA, Ochroch EA. Comparison between the analgesic efficacy of transversus abdominis plane (TAP) block and placebo in open retropubic radical prostatectomy: a prospective, randomized, double-blinded study. J Clin Anesth 2013; 25: 459-65.

49. McDonnell JG, Curley G, Carney J, Benton A, Costello J, Maharaj $\mathrm{CH}$, et al. The analgesic efficacy of transversus abdominis plane block after cesarean delivery: a randomized controlled trial.
Anesth Analg 2008; 106: 186-91.

50. McDonnell JG, O’Donnell B, Curley G, Heffernan A, Power C, Laffey JG. The analgesic efficacy of transversus abdominis plane block after abdominal surgery: a prospective randomized controlled trial. Anesth Analg 2007; 104: 193-7.

51. Abrahams M, Derby R, Horn JL. Update on ultrasound for truncal blocks: a review of the evidence. Reg Anesth Pain Med 2016; 41: 275-88.

52. Lancaster P, Chadwick M. Liver trauma secondary to ultrasoundguided transversus abdominis plane block. Br J Anaesth 2010; 104: 509-10.

53. Naidu RK, Richebe P. Probable local anesthetic systemic toxicity in a postpartum patient with acute Fatty liver of pregnancy after a transversus abdominis plane block. A A Case Rep 2013; 1: 72-4.

54. Ferguson S, Thomas V, Lewis I. The rectus sheath block in paediatric anaesthesia: new indications for an old technique? Paediatr Anaesth 1996; 6: 463-6.

55. Willschke H, Bosenberg A, Marhofer P, Johnston S, Kettner SC, Wanzel O, et al. Ultrasonography-guided rectus sheath block in paediatric anaesthesia--a new approach to an old technique. $\mathrm{Br}$ J Anaesth 2006; 97: 244-9.

56. Gurnaney HG, Maxwell LG, Kraemer FW, Goebel T, Nance ML, Ganesh A. Prospective randomized observer-blinded study comparing the analgesic efficacy of ultrasound-guided rectus sheath block and local anaesthetic infiltration for umbilical hernia repair. Br J Anaesth 2011; 107: 790-5.

57. Takebayashi K, Matsumura M, Kawai Y, Hoashi T, Katsura N, Fukuda S, et al. Efficacy of transversus abdominis plane block and rectus sheath block in laparoscopic inguinal hernia surgery. Int Surg 2015; 100: 666-71.

58. Bakshi SG, Mapari A, Shylasree TS. REctus Sheath block for postoperative analgesia in gynecological ONcology Surgery (RESONS): a randomized-controlled trial. Can J Anaesth 2016; 63: 1335-44.

59. Dingeman RS, Barus LM, Chung HK, Clendenin DJ, Lee CS, Tracy $\mathrm{S}$, et al. Ultrasonography-guided bilateral rectus sheath block vs local anesthetic infiltration after pediatric umbilical hernia repair: a prospective randomized clinical trial. JAMA Surg 2013; 148: 707-13.

60. Bashandy GM, Elkholy AH. Reducing postoperative opioid consumption by adding an ultrasound-guided rectus sheath block to multimodal analgesia for abdominal cancer surgery with midline incision. Anesth Pain Med 2014; 4: e18263.

61. Purdy M, Kinnunen M, Kokki M, Anttila M, Eskelinen M, Hautajarvi $\mathrm{H}$, et al. A prospective, randomized, open label, controlled study investigating the efficiency and safety of 3 different methods of rectus sheath block analgesia following midline laparoto- 
my. Medicine (Baltimore) 2018; 97: e9968.

62. Eldawlatly AA, Aldohayan A. Combined transversus abdominis plane block and rectus sheath block in laparoscopic peritoneal dialysis catheter insertion. Saudi J Anaesth 2016; 10: 251-2.

63. Randhawa K, Soumian S, Kyi M, Khaira H. Sonographic assessment of the conventional 'blind' ilioinguinal block. Can J Anaesth 2010; 57: 94-5.

64. Khedkar SM, Bhalerao PM, Yemul-Golhar SR, Kelkar KV. Ultrasound-guided ilioinguinal and iliohypogastric nerve block, a comparison with the conventional technique: an observational study. Saudi J Anaesth 2015; 9: 293-7.

65. Schmutz M, Schumacher PM, Luyet C, Curatolo M, Eichenberger U. Ilioinguinal and iliohypogastric nerves cannot be selectively blocked by using ultrasound guidance: a volunteer study. Br J Anaesth 2013; 111: 264-70.

66. Willschke H, Bösenberg A, Marhofer P, Johnston S, Kettner S, Eichenberger U, et al. Ultrasonographic-guided ilioinguinal/ iliohypogastric nerve block in pediatric anesthesia: what is the optimal volume? Anesth Analg 2006; 102: 1680-4.

67. Willschke H, Marhofer P, Bösenberg A, Johnston S, Wanzel O, Cox SG, et al. Ultrasonography for ilioinguinal/iliohypogastric nerve blocks in children. Br J Anaesth 2005; 95: 226-30.

68. Beaussier M, Weickmans H, Abdelhalim Z, Lienhart A. Inguinal herniorrhaphy under monitored anesthesia care with ilioinguinal-iliohypogastric block: the impact of adding clonidine to ropivacaine. Anesth Analg 2005; 101: 1659-62.

69. Bell EA, Jones BP, Olufolabi AJ, Dexter F, Phillips-Bute B, Greengrass RA, et al. Iliohypogastric-ilioinguinal peripheral nerve block for post-Cesarean delivery analgesia decreases morphine use but not opioid-related side effects. Can J Anaesth 2002; 49: 694-700.

70. Oriola F, Toque Y, Mary A, Gagneur O, Beloucif S, Dupont H. Bilateral ilioinguinal nerve block decreases morphine consumption in female patients undergoing nonlaparoscopic gynecologic surgery. Anesth Analg 2007; 104: 731-4.

71. Carney J, Finnerty O, Rauf J, Bergin D, Laffey JG, McDonnell JG. Studies on the spread of local anaesthetic solution in transversus abdominis plane blocks. Anaesthesia 2011; 66: 1023-30.

72. Ueshima H, Otake H, Lin JA. Ultrasound-guided quadratus lumborum block: an updated review of anatomy and techniques. Biomed Res Int 2017; 2017: 2752876.

73. Willard FH, Vleeming A, Schuenke MD, Danneels L, Schleip R. The thoracolumbar fascia: anatomy, function and clinical considerations. J Anat 2012; 221: 507-36.

74. El-Boghdadly K, Elsharkawy H, Short A, Chin KJ. Quadratus lumborum block nomenclature and anatomical considerations. Reg Anesth Pain Med 2016; 41: 548-9.

75. Elsharkawy H, El-Boghdadly K, Kolli S, Esa WAS, DeGrande S, Soliman LM, et al. Injectate spread following anterior sub-costal and posterior approaches to the quadratus lumborum block: a comparative cadaveric study. Eur J Anaesthesiol 2017; 34: 58795.

76. Carline L, McLeod GA, Lamb C. A cadaver study comparing spread of dye and nerve involvement after three different quadratus lumborum blocks. Br J Anaesth 2016; 117: 387-94.

77. Blanco R, Ansari T, Girgis E. Quadratus lumborum block for postoperative pain after caesarean section: a randomised controlled trial. Eur J Anaesthesiol 2015; 32: 812-8.

78. Blanco R, Ansari T, Riad W, Shetty N. Quadratus lumborum block versus transversus abdominis plane block for postoperative pain after cesarean delivery: a randomized controlled trial. Reg Anesth Pain Med 2016; 41: 757-62.

79. Forero M, Adhikary SD, Lopez H, Tsui C, Chin KJ. The erector spinae plane block: a novel analgesic technique in thoracic neuropathic pain. Reg Anesth Pain Med 2016; 41: 621-7.

80. Chin KJ, Adhikary S, Sarwani N, Forero M. The analgesic efficacy of pre-operative bilateral erector spinae plane (ESP) blocks in patients having ventral hernia repair. Anaesthesia 2017; 72: 45260.

81. Sá M, Graça R, Reis H, Cardoso JM, Sampaio J, Pinheiro C, et al. Superior gluteal nerve: a new block on the block? Rev Bras Anestesiol. 2017. doi: 10.1016/j.bjan.2016.11.001. [Epub ahead of print]. 